\title{
Galaxy identifications in the ROSAT All-Sky Survey`
}

\author{
H.-U. Zimmermann, Th. Boller, S. Döbereiner, and W. Pietsch \\ Max-Planck-Institut für Extraterrestrische Physik, Postfach 1312, 85471 Garching, Germany \\ Received 5 February 2001 / Accepted 3 August 2001

\begin{abstract}
In a correlation study of the ROSAT All-Sky Survey Bright Source Catalogue (RASS-BSC) with counterparts within a search radius of 100 arcsec. A visual screening process was applied to classify the reliability of the correlations. 547 correlations have been quoted as reliable identifications. From these, 349 sources are known as active galaxies. Although for the other sources no hints for activity were found in the literature, $69 \%$ of those for which we have distances show X-ray luminosities exceeding those of normal galaxies, a clear sign that these galaxies also own hitherto unreported X-ray active components. Some objects are located inside or in the direction of a known group or cluster of galaxies. Their X-ray flux may therefore be in part affected by hot gas emission. Luminosity and $\log N-\log S$ distributions are used to characterize different subsamples. Nuclei that are both optically and X-ray active are found predominantly in spirals. Two special source samples are defined, one with candidates for X-ray emission from hitherto unknown groups or clusters of galaxies, and one with high $\mathrm{X}$-ray luminosity sources, that are likely candidates to possess hitherto unreported active galactic nuclei. Besides a compilation of X-ray and optical parameters, also X-ray overlays on optical images for all the objects are supplied as part of this work.
\end{abstract} \\ the Catalogue of Principal Galaxies (PGC) 904 X-ray sources were found that possess possible extragalactic
}

Key words. surveys - X-rays - galaxies: general

\section{Introduction}

In 1990/91 the ROSAT observatory, using the PSPC detector in the focus of the X-ray telescope, scanned the whole sky during a half year period. It established the first all sky survey in the X-ray regime with an imaging telescope (Trümper 1983). Using a maximum likelihood source detection algorithm (Cruddace et al. 1987; Boese \& Döbereiner 2001) about 80000 sources with a likelihood $\geq 10$ have been found in the sky images of the ROSAT All-Sky Survey (RASS). Different attempts are underway to identify parts of this large data base with counterparts in other wavelength regimes to enable investigations of the broad band properties of different astronomical object classes.

This paper reports identification results obtained during the preparation of a study on the "X-ray properties of normal galaxies". From observations with the EINSTEIN observatory it is known that the X-ray emission of normal galaxies is on the order of $10^{38}$ to $10^{42} \mathrm{erg} \mathrm{s}^{-1}$ (1989). This

Send offprint requests to: H.-U. Zimmermann,

e-mail: zim@mpe.mpg.de

* Full data set (Table 1) and the 904 overlay images are only available at CDS via anonymous ftp to cdsarc.u-strasbg.fr $(130.79 .128 .5)$ or via

http://cdsweb.u-strasbg.fr/cgi-bin/qcat?J/A+A/378/30 should allow to detect them in the ROSAT All-Sky Survey data with typical exposures of a few hundred seconds up to distances of $100 \mathrm{Mpc}$.

Galaxies cannot be identified in the RASS data solely by their X-ray signature. We therefore had to correlate RASS sources with an optical database comprising most of the galaxies in the relevant distance regime. After different attempts we finally concluded that the Catalogue of Principal Galaxies (Paturel et al. 1989), updated by the NASA/IPAC Extragalactic Database (NED), best fulfilled our purposes for a rather complete sample of the objects we are interested in the study.

\section{The correlation samples}

\subsection{The X-ray data base}

For the study it is important to include only objects where the X-ray data promise high reliability for the existence of the source and allow some confidence in the derived positions and intensities. We found that the selection criteria, as applied for the compilation of the ROSAT All-Sky Survey Bright Source Catalogue (RASSBSC) (Voges et al. 1999), are a reasonable basis for our work. 


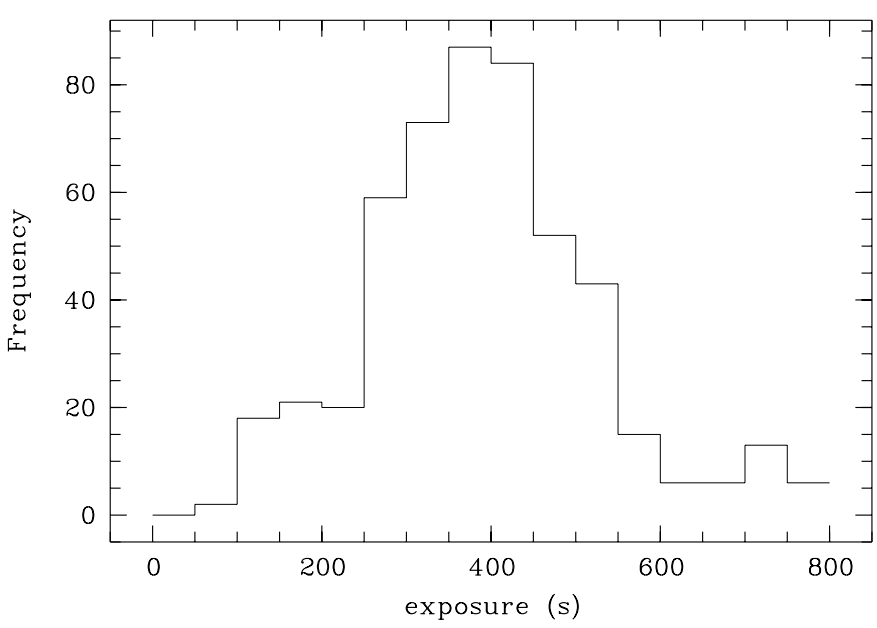

Fig. 1. Distribution of exposure times for the X-ray/PGC correlation sample (547 objects). Not shown are $8 \%$ of the observations with exposure times $>800 \mathrm{~s}$.

The RASS-BSC is a subset of the RASS database comprising $18811 \mathrm{X}$-ray sources selected from the whole sample by the following criteria:

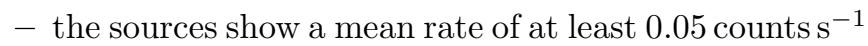
in the $0.1-2.4 \mathrm{keV}$ energy band during the RASS observations;

- the sources were detected with a likelihood $\geq 15$;

- from each source at least 15 counts were collected.

At a brightness limit of 0.1 counts $^{-1}$ the sky coverage of the BSC is complete at a level of $92 \%$.

The width of the effective survey point spread function ( $F W H M$ about 45 arcsec) and the use of a maximum likelihood (ML) detection algorithm (Boese \& Döbereiner 2001; Cruddace et al. 1987), delivering best estimates for point-like sources, limit the reliability of the derived source properties in cases where 2 or more X-ray sources are within the acceptance window used by the algorithm (typically a circle of $300 \mathrm{arcsec}$ diameter) and in cases where X-ray sources are clearly extended. The authors of the BSC paper demonstrated that $90 \%$ of point-like X-ray sources (stars) have a positional accuracy of $\leq 25$ arcsec.

The exposure times of the objects in the final X-ray/PGC correlation sample range from 56 to $17165 \mathrm{~s}$, with $79 \%$ of the sources having exposures between 200 and 600 s (see Fig. 1).

Fluxes in the ROSAT band have been calculated using the EXSAS software (Zimmermann et al. 1997). The spectral parameters have been taken from ROSAT observations of extragalactic sources; a power law with photon index 2.3 (Hasinger et al. 1991; Walter \& Fink 1993) is taken using the galactic absorbing column density $N_{\mathrm{H}}$ (Dickey \& Lockman 1990) along the line of sight. Fluxes of nearby galaxies may be lower limit values, if the $\mathrm{X}$-ray emission is extended. The sources in our final sample possess measured fluxes between $6 \times 10^{-13}$ and $7 \times$ $10^{-10} \mathrm{erg} \mathrm{cm}^{-2} \mathrm{~s}^{-1}$. A galaxy with $10^{42} \mathrm{erg} \mathrm{s}^{-1}$ at $100 \mathrm{Mpc}$

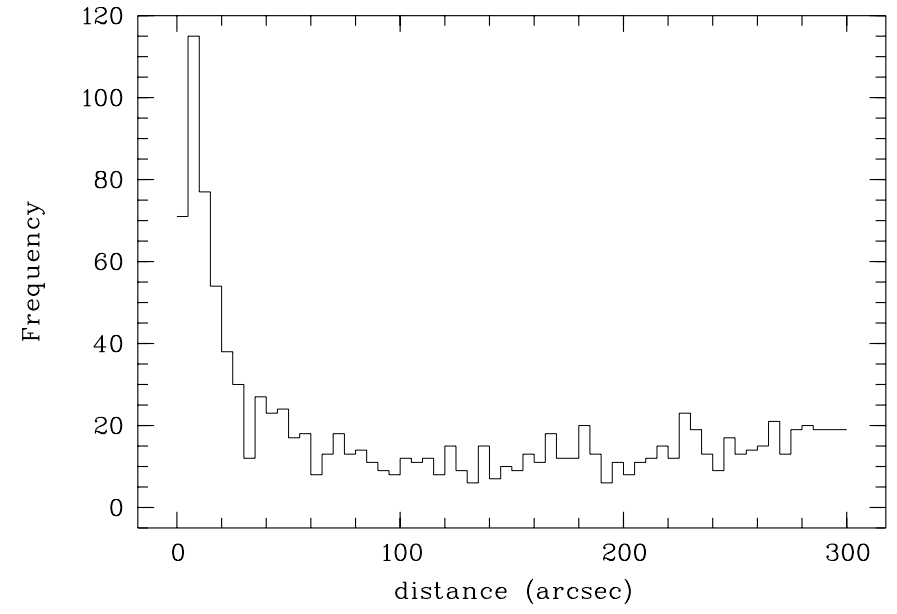

Fig. 2. Distribution of distances between objects in the PGC and the BSC catalogs for search radii up to 300 arcsec.

distance delivers a flux of about $8 \times 10^{-13} \mathrm{erg} \mathrm{cm}^{-2} \mathrm{~s}^{-1}$ and at least $300 \mathrm{~s}$ of exposure are necessary to detect it as a source that would find its way into the RASS-BSC.

\subsection{The optical data base}

The Catalog of Principal galaxies (Paturel et al. 1989) was compiled in the 1980's from different catalogs of extragalactic objects (galaxies) and comprises in total 73197 sources. By construction it contains most of the nearby extragalactic objects, but is less complete in distant and fainter objects.

Since the compilation of the PGC, for many individual galaxies updated information on some of the galaxy parameters became available. This is especially true for the positions of individual galaxies, where the accuracy in the original PGC often presents a major problem for unique correlations. We therefore decided to base the optical galaxy sample on the PGC objects but, for each individual object, rely on the parameters as given in the NASA Extragalactic Database (NED). The other possibility, to start directly from the NED data base, had also been considered. While the number of positional correlations, $\sim 4418$ within a search radius of 100 arcsec, is about a factor of 4 higher than with the PGC, the number of normal galaxies (not known to be active) is only marginally larger, as was found by checks. Due to the effort in screening visually 4 times more correlations while not detecting many more of these objects, we stayed with the PGC membership as the main galaxy selection criterium.

For 61 sources of our sample we performed optical observations (Pietsch et al. 1998; Bischoff et al. 1999) to better evaluate the position (accuracy $<2$ arcsec) and classification of these objects. The optical observations will be continued in future projects.

\section{The correlation process}

To establish a reasonable search radius around the X-ray sources within which to look for counterparts in the PGC, 


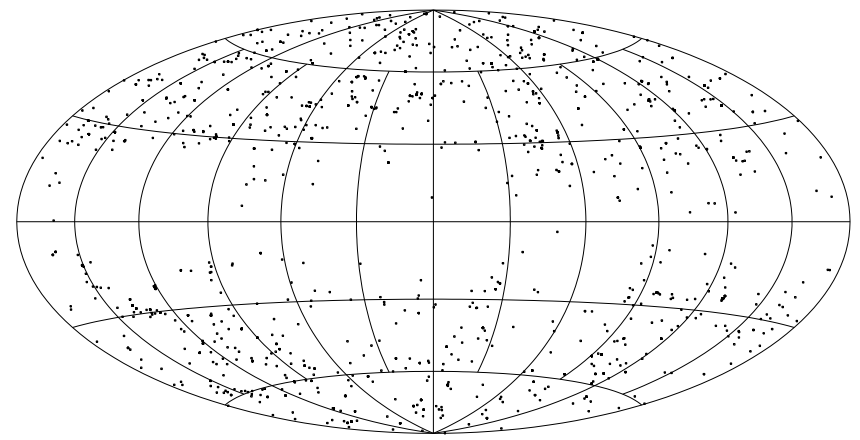

Fig. 3. Sky distribution of the $904 \mathrm{X}$-ray sources in our sample in galactic coordinates. Galactic longitude starts at the center. The lack of galaxies near the galactic plane is due to high absorption in our galaxy.

we first determined the number of correlations per radius interval between the 2 data bases as a function of the search radius (Fig. 2).

In such a representation the true correlations show up as a peak near small radii, while chance correlations between the two catalogs produce the right-hand part of the profile that is slowly rising with increasing radius. The width of the initial peak is broadened mainly due to positional errors in the two data bases. $90 \%$ of the BSC X-ray point sources have a mean position error of $\leq 25$ arcsec, while the PGC catalog does not provide explicit position errors. In many cases they were found to be appreciable larger. Additional broadening may arise when the maximum of the X-ray flux originates at positions off the optical center of the galaxy. Also the optical center of irregular nearby galaxies is not always easily determined.

To include the vast majority of true correlations and at the same time accept only a small number of chance coincidences we chose a search radius of 100 arcsec, at the minimum of the distribution in Fig. 2. From the figure we estimate that up to $7 \%$ of real correlations are missed by that choice while the number of chance coincidences (before screening) is about $12 \%$.

The working sample is defined by 904 X-ray sources from the BSC surrounded by 1124 objects from the PGC lying within a radius of 100 arcsec of the X-ray sources. The reduced spatial resolution in the X-ray data of this survey prevents in many cases to resolve multiple galaxy systems into their individual components.

Figure 3 shows the all sky distribution of the X-ray sources in the investigated sample.

\section{Data screening and verification}

Besides chance coincidences there may be other reasons that can put a correlation in question. In a further step we therefore tried to identify which of the correlation pairs are real, that is, which of the galaxies are also the sources of the observed X-ray emission.

For this purpose we overlaid X-ray contours smoothed by a 45 arcsec Gaussian on an optical image (POSS plates, see acknowledgements) of about 8.5 arcmin $\times 8.5$ arcmin size centered on the position of the X-ray source (see examples in Figs. 4 to 7 ).

In these images the original positions of the PGC sources and additionally all sources from the NED database up to a distance of 3 arcmin around the X-ray counterpart are marked by white squares (PGC) and circles (NED) as well as the center of the X-ray emission (cross) as derived by the maximum likelihood process. Besides the appearance of the X-ray contours and the distance to the optical counterpart, also the position and object properties of the other sources in the field, like known activity or the membership in a group or cluster of galaxies, entered the qualification process.

The reliability of the correlation was estimated and expressed by the following 4 terms: no, questionable, good, and very good.

This classification scheme must be regarded as a first approach in determining whether the observed X-ray emission originates from the optical galaxy in question. A reliable answer is sometimes difficult or even impossible. Part of the problem arises from the X-ray data itself: often the X-ray contours originate from little more than a dozen photons, leading to poorly determined positions and contours. But also the optical positions of the NED report for 86 of the 1124 optical objects uncertainties between 30 and 300 arcsec, making it sometimes difficult to identify uniquely the objects in the optical images.

Specific difficulties were encountered when the X-ray emission came from the direction of a known or suspected group or cluster of galaxies. From the X-ray data it is usually not possible to decide how much of the X-rays stem from the hot cluster gas and how much can be attributed to a single galaxy. Many clusters contain a dominant CD galaxy in their gravitational center, that forms also the $\mathrm{X}$-ray emission center of the hot cluster gas.

On the other hand, if statistics are good enough, the $\mathrm{X}$-ray contours from known clusters of galaxies show characteristics, that allow in some cases a recognition of these extended structures also in images where no explicit hints for the presence of a group or cluster of galaxies have been found in the literature. The source extent parameter, calculated by the ML source algorithm, in many cases gives first hints to these sources. In the main table we have marked all those X-ray sources, in the neighbourhood of which ( $\leq 3$ arcmin) no group or cluster of galaxies are reported (NED), but where the X-ray contours indicate relevant features. In addition to the contours also optical field properties entered the decision process (e.g. type of galaxy counterpart, no obvious counterpart, high density of galaxies, X-ray emission shifted, etc.).

In the case of narrow galaxy pairs or triplets, that were not resolved by ROSAT, we marked only one member, the most probable one, as the identified counterpart.

To give the reader a feeling of the considerations involved in the screening, Figs. 4 to 7 show some typical examples for the 4 reliability levels, including the principal lines of reasoning in the figure captions. 


\begin{tabular}{|c|c|c|c|c|c|c|c|c|c|c|c|c|c|c|}
\hline SEQU & $\begin{array}{l}\text { xid } \\
\text { sid }\end{array}$ & $\begin{array}{c}\operatorname{name}_{-} x \\
\operatorname{RADEC} x \\
\end{array}$ & $\begin{array}{l}\text { exposure } \\
\text { pos_err_x }\end{array}$ & $\begin{array}{l}\text { rate } \\
\text { rate_err }\end{array}$ & $\begin{array}{l}\text { lh } \\
\text { counts }\end{array}$ & $\begin{array}{l}\text { ext_lh } \\
\text { extent }\end{array}$ & $\begin{array}{l}\text { name_opt } \\
\text { RADEC_opt }\end{array}$ & $\begin{array}{l}\text { mag } \\
\text { pos_err }\end{array}$ & $\begin{array}{l}\text { type } \\
\text { type } 1 \\
\end{array}$ & $\begin{array}{l}\text { activity } \\
\text { velocity }\end{array}$ & $\begin{array}{l}\text { or } \\
\mathrm{cl}\end{array}$ & $\begin{array}{l}\mathrm{NH} \\
\text { dist }\end{array}$ & $\begin{array}{l}\log F 1 u x x \\
\log \_x\end{array}$ & $\begin{array}{l}\text { correlation } \\
\text { comment }\end{array}$ \\
\hline 4 & $\begin{array}{l}4 \\
1\end{array}$ & 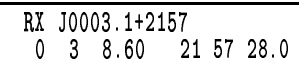 & $\begin{array}{r}293 \\
13\end{array}$ & $\begin{array}{l}0.09642 \\
0.01970\end{array}$ & $\begin{array}{r}57 \\
28.3\end{array}$ & $\begin{array}{l}0 \\
0\end{array}$ & 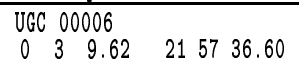 & $\begin{array}{r}14.38 \\
0.3\end{array}$ & $\begin{array}{l}G \\
P A C\end{array}$ & $\begin{array}{r}\text { Sy1.8 } \\
6582 .\end{array}$ & $\begin{array}{l}2 \\
0\end{array}$ & $\begin{array}{r}0.443 \\
16.6\end{array}$ & $\begin{array}{r}-11.59 \\
42.43\end{array}$ & good \\
\hline 7 & $\begin{array}{l}7 \\
1\end{array}$ & 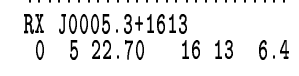 & $\begin{array}{r}504 \\
14\end{array}$ & $\begin{array}{l}0.07616 \\
0.01464\end{array}$ & $\begin{array}{r}49 \\
38.4\end{array}$ & $\begin{array}{l}0 \\
0\end{array}$ & 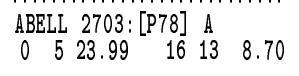 & 7.5 & G & 34920 . & $\begin{array}{l}3 \\
3\end{array}$ & $\begin{array}{r}0.368 \\
18.7\end{array}$ & $\begin{array}{r}-11.75 \\
43.73\end{array}$ & questionable \\
\hline 10 & $\begin{array}{r}10 \\
1\end{array}$ & 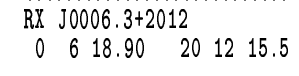 & $\begin{array}{r}269 \\
7\end{array}$ & $\begin{array}{l}2.48200 \\
0.09737\end{array}$ & $\begin{array}{r}2710 \\
667.7\end{array}$ & $\begin{array}{l}27 \\
17\end{array}$ & 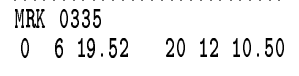 & $\begin{array}{r}13.75 \\
0.3\end{array}$ & $\begin{array}{l}\text { UvES } \\
\text { SO/a }\end{array}$ & $\begin{array}{l}\text { Sy1 } \\
\\
\end{array}$ & $\begin{array}{l}2 \\
0\end{array}$ & $\begin{array}{r}0.396 \\
10.1\end{array}$ & $\begin{array}{r}-10.21 \\
43.94\end{array}$ & very good \\
\hline 14 & $\begin{array}{r}14 \\
1\end{array}$ & 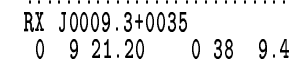 & $\begin{array}{r}394 \\
10\end{array}$ & $\begin{array}{l}0.11360 \\
0.01893\end{array}$ & $\begin{array}{r}76 \\
44.8\end{array}$ & $\begin{array}{r}1 \\
10\end{array}$ & 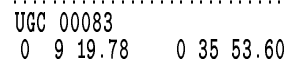 & $\begin{array}{r}16.5 \\
5.0\end{array}$ & $\stackrel{G}{\mathrm{Sb}}$ & & $\begin{array}{l}2 \\
0\end{array}$ & $\begin{array}{l}0.295 \\
137.5\end{array}$ & -11.64 & $\begin{array}{l}\text { no } \\
\text { Xrays from star }\end{array}$ \\
\hline 16 & $\begin{array}{r}16 \\
1\end{array}$ & $\begin{array}{l}\mathrm{RX} J 0010.1-0442 \\
01010.20-44224.9\end{array}$ & $\begin{array}{r}356 \\
11\end{array}$ & $\begin{array}{l}0.22120 \\
0.02790\end{array}$ & $\begin{array}{r}150 \\
78.7\end{array}$ & $\begin{array}{r}6 \\
21\end{array}$ & $\begin{array}{l}\text { MCG }-01-01-043 \\
01010.04-44237.50\end{array}$ & $\begin{array}{r}16.0 \\
5.0\end{array}$ & $\begin{array}{l}\text { G } \\
P e c\end{array}$ & $\begin{array}{ll}\text { Sy2 } & \\
& 8851 .\end{array}$ & $\begin{array}{l}2 \\
0\end{array}$ & $\begin{array}{r}0.313 \\
12.7\end{array}$ & $\begin{array}{r}-11.33 \\
42.95\end{array}$ & very good \\
\hline 30 & $\begin{array}{r}26 \\
1\end{array}$ & 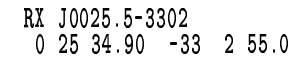 & $\begin{array}{r}330 \\
11\end{array}$ & $\begin{array}{l}0.24980 \\
0.03095\end{array}$ & $\begin{array}{r}134 \\
82.4\end{array}$ & $\begin{array}{l}0 \\
0\end{array}$ & 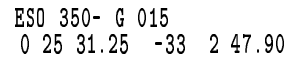 & $\begin{array}{r}14.23 \\
1.0\end{array}$ & $\begin{array}{l}G \\
E+3\end{array}$ & 14949. & $\begin{array}{l}2 \\
3\end{array}$ & $\begin{array}{r}0.169 \\
46.4\end{array}$ & -11.46 & no \\
\hline 48 & $\begin{array}{r}38 \\
1\end{array}$ & 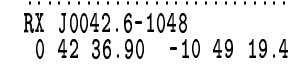 & $\begin{array}{r}365 \\
8\end{array}$ & $\begin{array}{l}0.24440 \\
0.02782\end{array}$ & $\begin{array}{r}240 \\
89.2\end{array}$ & $\begin{array}{l}1 \\
9\end{array}$ & 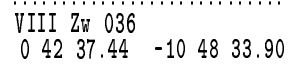 & $\begin{array}{r}14.6 \\
150.0\end{array}$ & $\stackrel{G}{\mathrm{E} 2}$ & $\begin{array}{l}\text { Sy } 1.5 \\
12390 .\end{array}$ & $\begin{array}{r}12 \\
0\end{array}$ & $\begin{array}{r}0.262 \\
46.3\end{array}$ & $\begin{array}{r}-11.34 \\
43.23\end{array}$ & good \\
\hline 89 & $\begin{array}{r}70 \\
1\end{array}$ & $\begin{array}{l}\text { RX J0116.1-1554 } \\
1 \quad 16 \quad 13.30-15 \quad 54 \quad 47.4\end{array}$ & $\begin{array}{r}396 \\
29\end{array}$ & $\begin{array}{l}0.13750 \\
0.02189\end{array}$ & $\begin{array}{r}37 \\
54.5\end{array}$ & $\begin{array}{l}14 \\
90\end{array}$ & 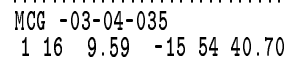 & $\begin{array}{r}16 \\
300.0\end{array}$ & $\ddot{G}$ & & $\begin{array}{l}2 \\
2\end{array}$ & $\begin{array}{r}0.169 \\
54.0\end{array}$ & -11.72 & questionable \\
\hline
\end{tabular}

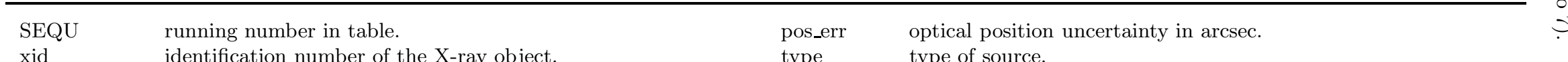

xid identification number of the X-ray object.

sid identifies the optical candidates for a specific $\mathrm{X}$-ray source.

type

type of source.

type1 detailed type of optical candidate.

name_x IAUC identifier for the $\mathrm{X}$-ray source.

RADEC_x RA and DEC positions (2000) of X-ray source.

activity

exposure exposure time of $\mathrm{X}$-ray source in $\mathrm{s}$.

velocity recession velocity in $\mathrm{km} / \mathrm{s}$.

pos_err_X uncertainty of X-ray position in arcsec.$$
\text { or }
$$

origin of parameters: 1 from PGC only, 2 from NED,

12 from NED + (Pietsch et al. 1998)

22 from NED + (Bischoff et al. 1999).

rate count rate of X-ray source in counts/s $(0.1-2.4 \mathrm{keV})$.

err_rate uncertainty of rate in counts/s.

$\mathrm{cl}$

th likelihood of detection.

counts total counts from X-ray source.

extlh likelihood that X-ray source is extended (interpret with care!). extent extent in arcsec

name_opt name of optical candidate

RADEC_opt RA and DEC positions of optical candidate.

mag $\quad B$ magnitude of optical candidate (NED).

$N_{\mathrm{H}}$

dist logFlux_x.

log_Lx.

correlation

comment whether in binary (1), group (2), or cluster of galaxies (3).

or line-of-sight projection near to cluster of galaxies (3).

galactic hydrogen column density in $10^{22} \mathrm{~cm}^{-2}$.

distance between X-ray object and optical candidate (arcsec).

$\log _{10}$ of X-ray flux in erg cm ${ }^{-2} \mathrm{~s}^{-1}$ in the $0.1-2.4 \mathrm{keV}$ band.

$\log _{10}$ of X-ray luminosity in erg $/ \mathrm{s}\left(H_{0}=70 \mathrm{~km} \mathrm{Mpc}^{-1} \mathrm{~s}^{-1}\right)$.

reliability of correlating the X-ray and optical source.

comments; cl? means source in suspected cluster sample. 

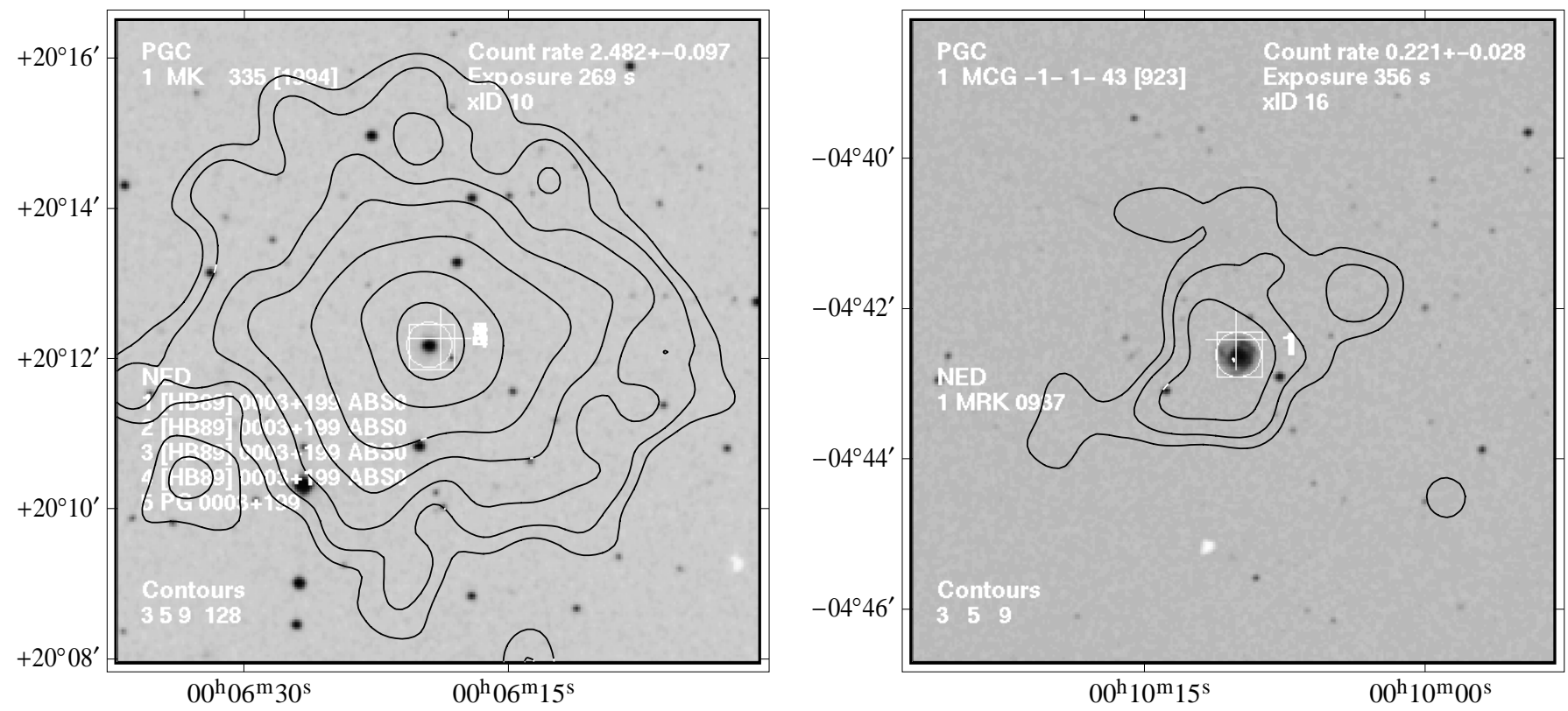

Fig. 4. Examples for the images used during the screening process. These 2 images represent examples with "very good" reliability: on the left an optically resolved Seyfert-1 object with well centered X-ray contours, on the right a Seyfert 2 object at similar space distance, with the expected smaller X-ray intensity. The cross indicates the X-ray position, squares the PGC positions and circles the NED positions. The numeric labels at the different positions point to the entries in the name lists displayed in the left part of the map. X-ray contour levels (in sigma above background) are denoted at the lower left corner. Count rate and exposure of the X-ray source are given, together with the identification number of the X-ray source (xID, from Col. 2 in Table 1), in the upper right corner.
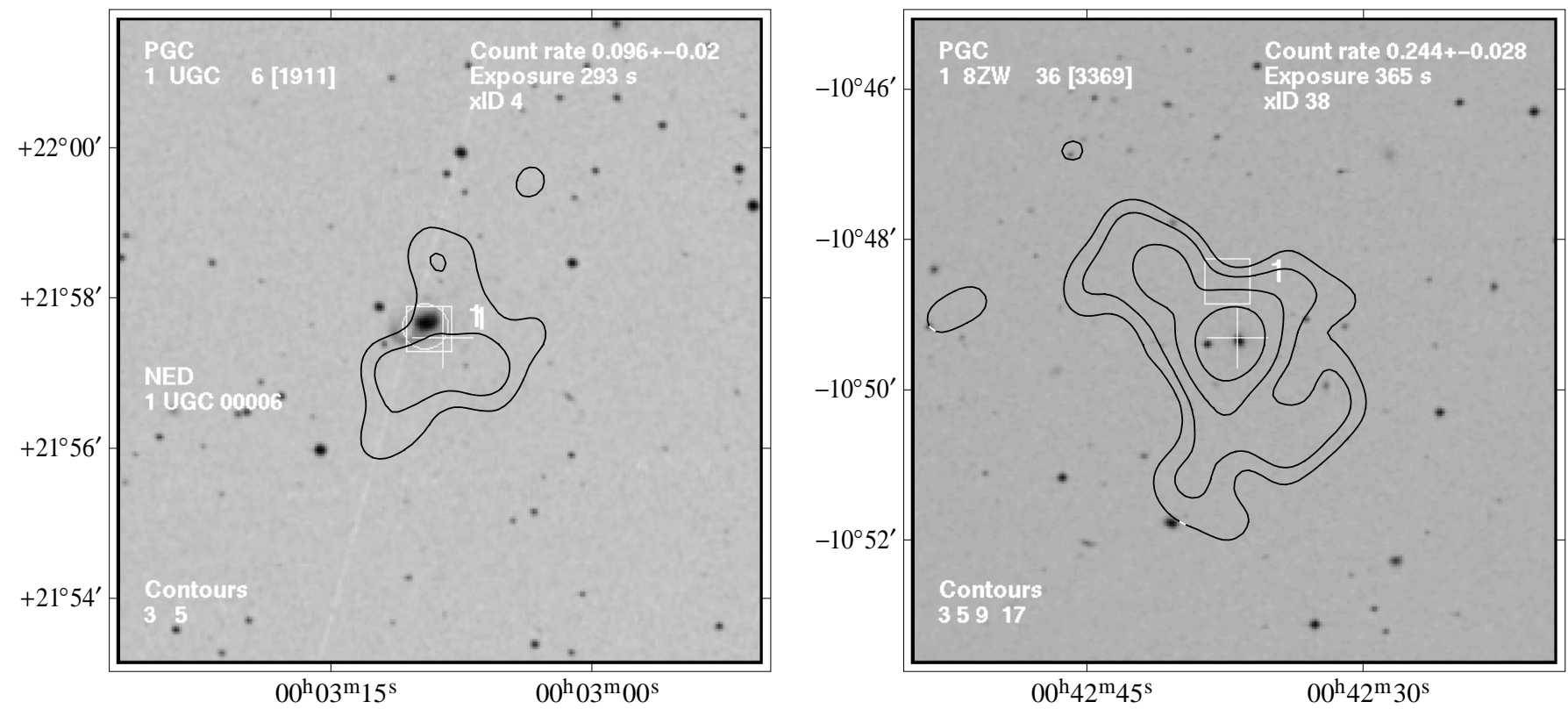

Fig. 5. The reliability of these 2 images is quoted as "good": on the left a 1.8 Seyfert, optically resolved, with poor X-ray contours, on the right an example, where the optical position of the Seyfert 1.5 object is off from the X-ray object by almost 50 arcsec, but where the optical position error is stated as 150 arcsec. The optical parameters (magnitude, extent) and the X-ray properties (contours, intensity) led us to accept the correlation as correct.

\section{Data tables and images}

Table 1 shows the structure and content of the sample list. The example entries in the table refer to the sources shown in Figs. 4 to 7 . The information on each source covers 2 lines. After 2 columns with entry identification follow 5 columns with X-ray parameters, specifying $\mathrm{X}$-ray name, position, exposure, rate and counts in the $0.1-2.4 \mathrm{keV}$ energy band, detection likelihood, and information on possible extent (mostly taken from BSC). 

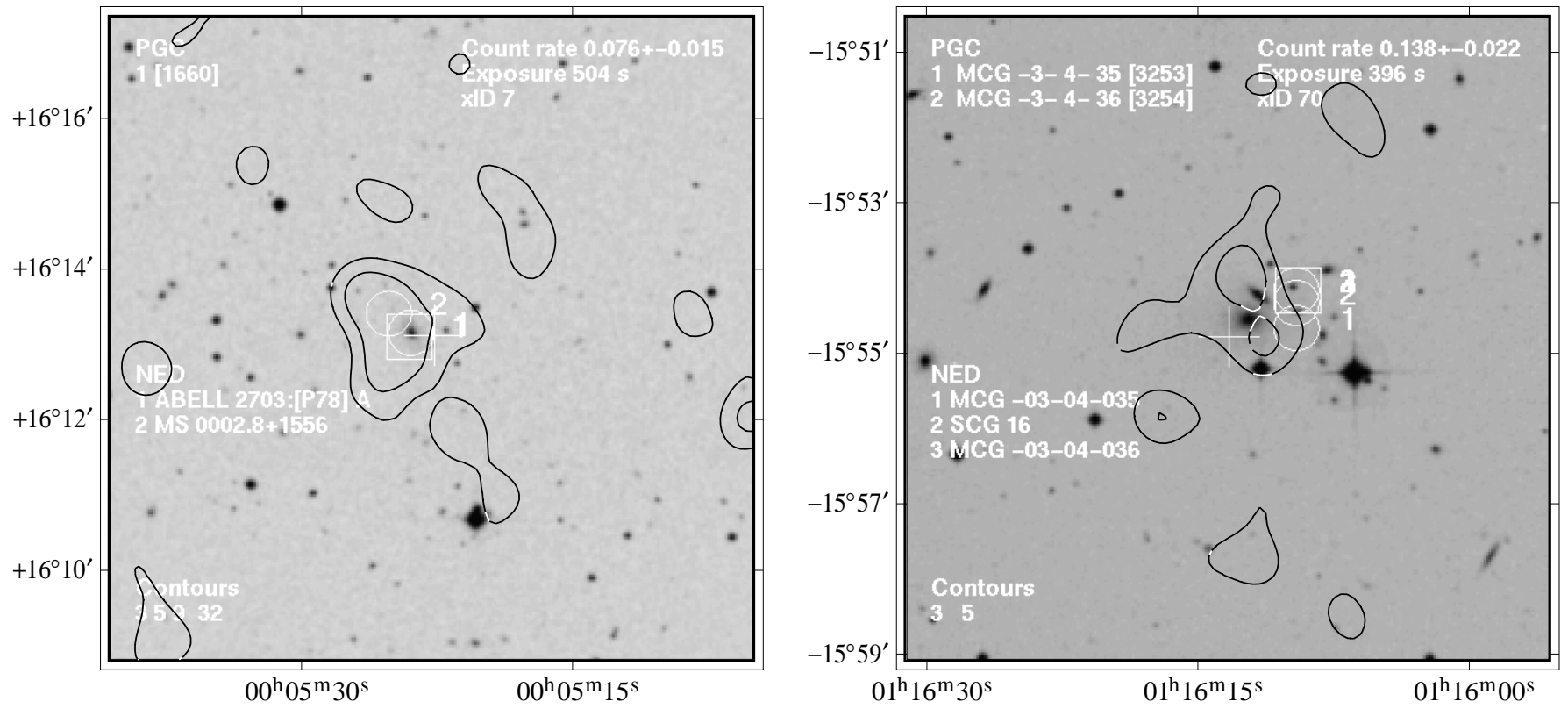

Fig. 6. 2 images that were quoted having questionable reliability: the left image shows X-ray contours that indicate extended hot gas, perhaps from the central cluster, while the right image is an example where we could not decide which of the optical galaxies is the probable counterpart and whether we see only hot gas in the galaxy group SCG 16 .
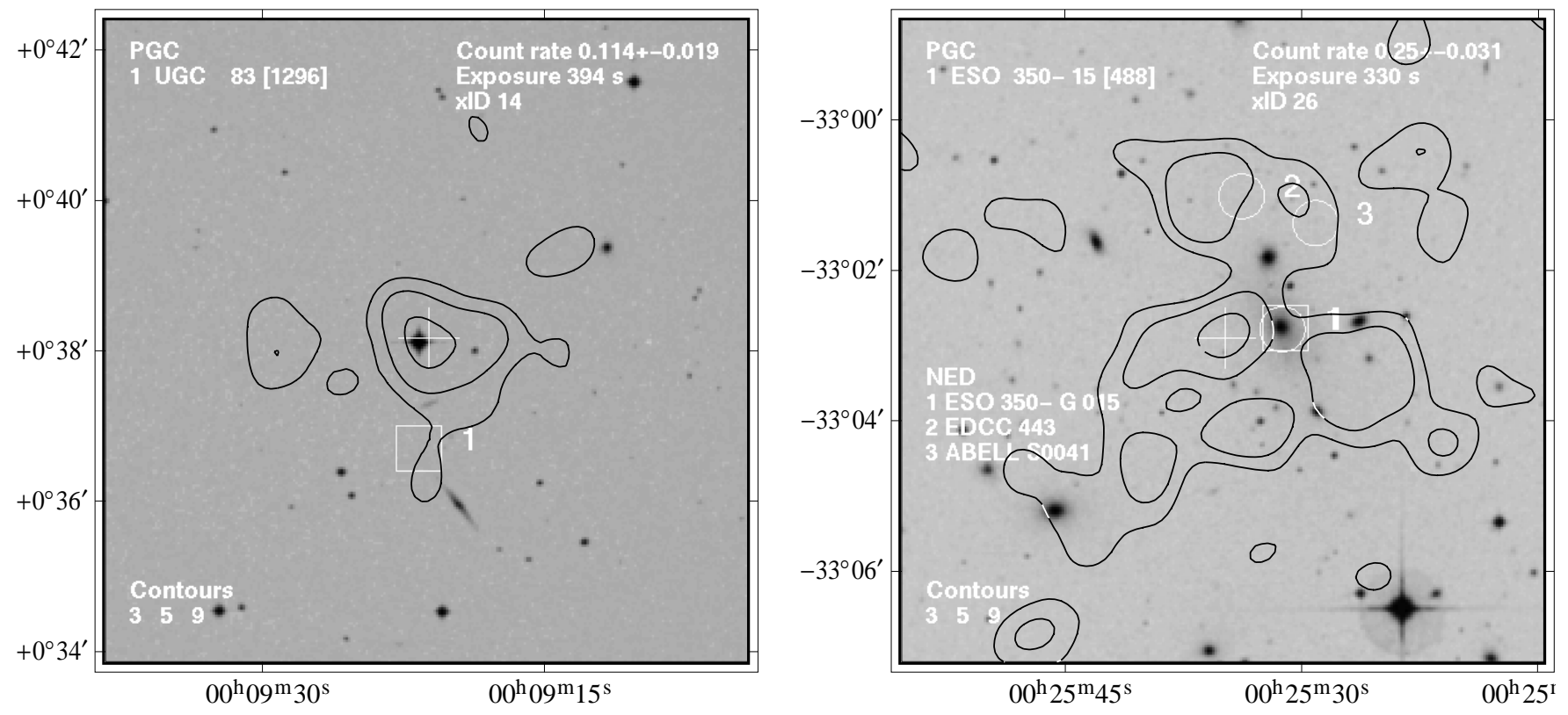

Fig. 7. 2 examples where a correlation was rejected: in the image on the left the counterpart due to its optical appearance is a star with no obvious galaxy connection, while the X-ray emission in the right image, looking at the contours, is dominated by hot cluster gas.

Then follow 5 columns with optical data, name, position, magnitude, galaxy type, activity, grouping info, and velocity (mostly taken from NED, in parts also from Pietsch et al. 1998; Bischoff et al. 1999). The last few columns contain an indicator of the data origin, the galactic absorbing column density towards the object (Dickey \& Lockman 1990), the distance between the $\mathrm{X}$-ray source and the optical candidate, the X-ray flux and luminosity, as well as the reliability of the correlation. Xray fluxes and luminosities have been calculated assuming a power law with photon index 2.3 (Hasinger et al. 1991; Walter \& Fink 1993), the relevant galactic absorbing column density $N_{\mathrm{H}}$ and, if available, the distance to the source. For some sources additional remarks are available in column comment, which contains also the membership to the suspected cluster sample (marked as cl?) mentioned in Sect. 4 on Data Screening.

The complete table, with 1124 correlation entries for the $904 \mathrm{X}$-ray sources, as well as the X-ray contour overlays on optical images for all of the $904 \mathrm{X}$-ray sources, 
is available in electronic form at the CDS via anonymous ftp to cdsarc.u-strasbg.fr (130.79.128.5) or via http://cdsweb.u-strasbg.fr/cgi-bin/qcat?J/A+A/378/30

\section{Discussion of the sample properties}

In the discussion we refer to the following sample designations:

- The complete sample comprising all $904 \mathrm{X}$-ray objects.

- A suspected cluster sample containing 101 X-ray objects.

- The reliable correlation sample containing the $547 \mathrm{X}$ ray objects with reliable correlation, on which the following subsamples are based.

- The candidate galaxies, 198 X-ray objects without reported activity, for which we discuss the properties of different subsamples.

- The active galaxies sample with 349 objects.

- A high X-ray luminosity sample comprising 23 objects, a subsample of the "candidate galaxies" with high correlation reliability.

As mentioned in Sect. 4 on Data Screening, we determined a suspected cluster sample, where the X-ray emission is supposed to originate, at least in part, from extended hot gas in a group or cluster of galaxies, but where no group or cluster of galaxies up to now has been reported in the literature (NED). This candidate list comprises 101 entries. 70 of these sources have been quoted as no valid or as questionable correlations, while in the rest most of the $\mathrm{X}$-ray emission was attributed to a single galaxy. These 101 objects form a subsample of likely candidates for Xray emission from groups and clusters of galaxies, worthwhile for further studies in the optical and X-ray band.

The reliability of the X-ray/PGC correlations for the 904 X-ray sources of the complete sample was denoted as "very good" for 367 objects, as "good" for 180, and as "questionable" for 161 objects. The remaining correlations were classified as being not real, that is they are either chance coincidences or their X-ray flux originates predominantly from extended emission of hot gas in galaxy groups or clusters.

The following discussion refers only to the 547 objects of the reliable correlation sample, that is, the objects having correlation reliabilities good or very good. These are grouped in 2 categories:

a) candidate galaxies, where the optical counterpart is not designated as LINER, Seyfert, QSO, or Blazar in the literature (mostly from NED). Our sample contains in total 198 such galaxies, of which 88 have been classified with reliability very good and 110 with reliability good;

b) active galaxies, mainly Seyferts, QSOs and a few LINERS and Blazars. This category comprises 349 objects (reliability very good 279 , good 70 ).

51 objects (36 of group a), 15 of group b)) lie inside or in direction to a known group or cluster of galaxies, but

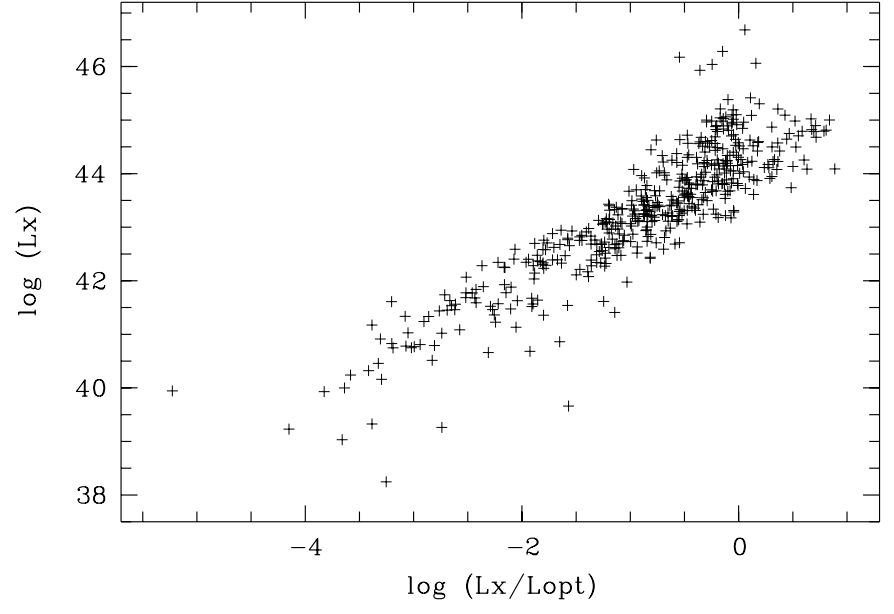

Fig. 8. X-ray luminosity versus the ratio of X-ray to optical luminosity (or flux) for sample objects with distance information.

display X-ray contours that give confidence that most of the observed X-ray emission is coming from the galaxy itself and not from an extended group or cluster gas component (checked by comparing the relevant $\log N-\log S$ distributions).

In the reliable correlation sample of 547 galaxies, for 488 objects we have distance information and therefore can calculate their X-ray and optical luminosities from the relevant fluxes, adopting a Hubble constant $H_{0}=$ $70 \mathrm{~km} \mathrm{Mpc}^{-1} \mathrm{~s}^{-1}$, which is in the range of values determined from the HST distance scale project (see e.g. Mould et al. 2000). Figure 8 shows the X-ray luminosity versus the ratio of X-ray to optical luminosities (or fluxes) in these objects, demonstrating the well-known correlation between optical and X-ray luminosities (see e.g. Fabbiano et al. 1992). Optical fluxes and luminosities have been derived from the optical $B$ magnitudines as provided by NED with no attempt to include bolometric corrections. The 6 objects at the high end of the X-ray luminosity scale are all quasars, $3 \mathrm{C} 273$ and the 5 objects in our sample with $1<z \leq 2$. All the other objects with known distances have $z<0.5$.

The optical activity as seen in Liners, Seyferts, QSOs, or Blazars originates from activity in the nucleus of the galaxy. While the high energy output of the nucleus, over many stages of absorption and reemission, finally deposits part of its initial energy in the observed optical output, the X-ray flux originates from regions closer to the central engine and therefore reflects generally more directly the activity in the central engine. In the optical regime therefore the nucleus usually contributes only indirectly to the overall output of the galaxy and absorption and galaxy geometry and aspect (with respect to the observer) play an important part. The X-ray flux from the nucleus, on the other hand, is the dominant part of the X-ray emission in the majority of X-ray active galaxies.

This is also reflected in the X-ray and optical luminosity distributions for the active galaxies and candidate 


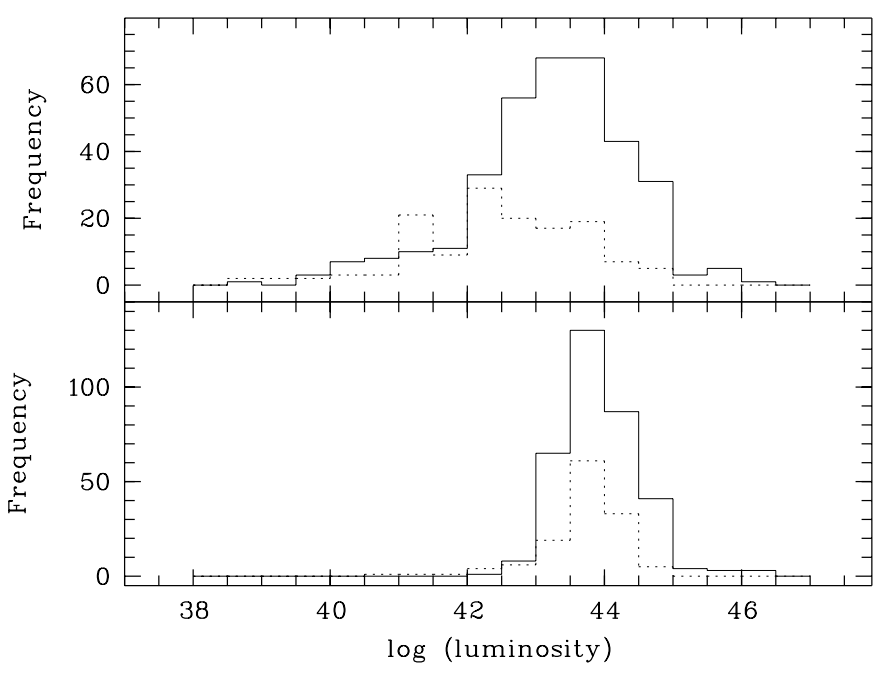

Fig. 9. X-ray luminosity distribution (upper panel) and optical luminosity distribution (lower panel) of galaxies having known distances in sample candidate galaxies (dotted line) and in sample active galaxies (full line).

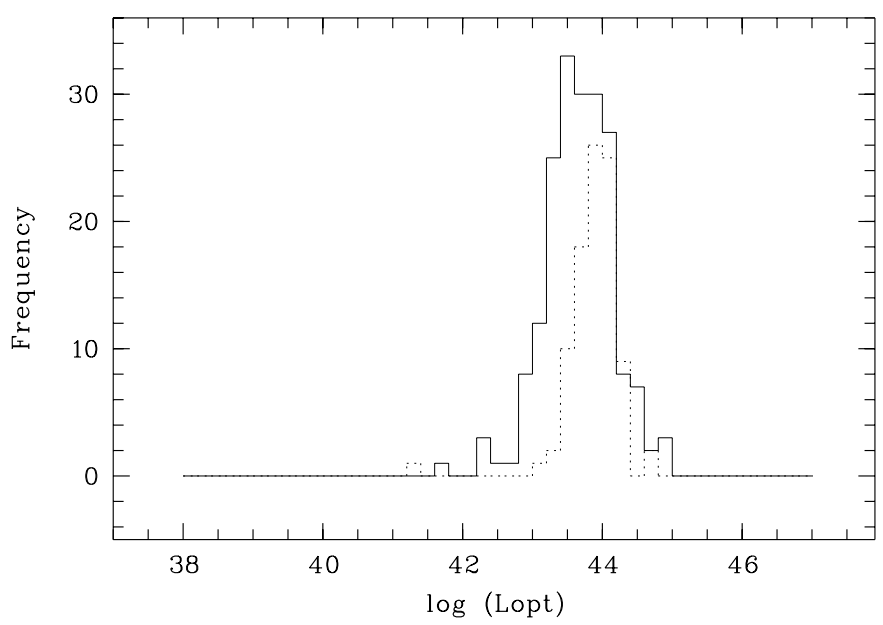

Fig. 10. Optical luminosity distribution of the spiral galaxies (full line) and the elliptical plus S0 galaxies (dashed line) independent of their activity status.

galaxies in our sample. Figure 9 shows in the upper panel the X-ray luminosity distribution for both the 198 candidate galaxies (dashed line) and the 349 active galaxies (full line). As one would expect the distribution of $a c$ tive galaxies is shifted towards higher X-ray luminosities in comparison to the candidate galaxies. The optical luminosity distribution (lower panel), on the other hand, does not show the same dependency (lower panel).

There is, however, as Fig. 10 shows, a difference in the width of the optical luminosity distribution between the spiral galaxies (full line) and the elliptical plus S0 galaxies (dashed line). The larger width of the distribution of spirals possibly derives from its broader scale in surface brightness due to the larger range of geometries compared to the elliptical plus S0 galaxy sample.

The $\log N-\log S$ distribution of the sample for both the X-ray and the optical flux is shown in Fig. 11.

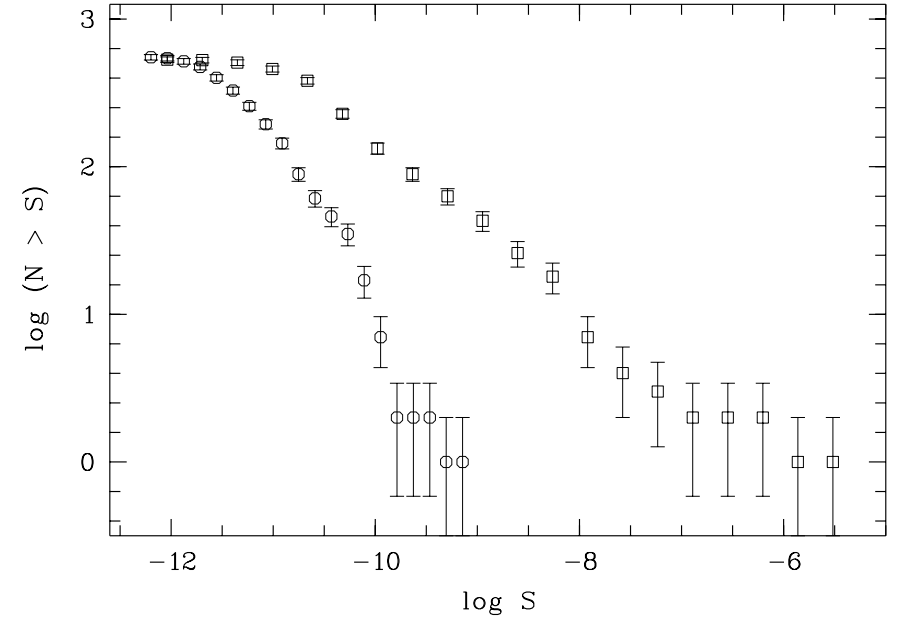

Fig. 11. $\log N-\log S$ distributions of the X-ray (circles) and optical (squares) flux $\mathrm{S}$ for 477 sample sources.

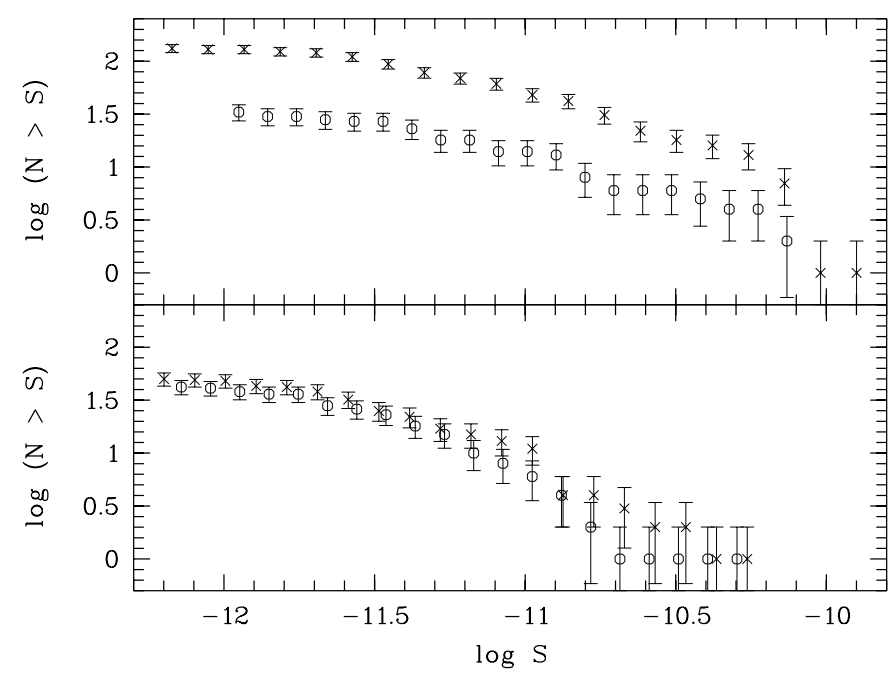

Fig. 12. $\log N-\log S$ distributions of the X-ray flux S for spirals (X) and elliptical plus S0 galaxies (circles), in the upper panel for the active galaxies sample, in the lower panel for the candidate galaxies. Optical activity is predominantly observed in spirals.

It demonstrates that the brightest $\mathrm{X}$-ray galaxies have been detected rather well, with a gradient near to -1.5 as expected for an Euclidian source distribution, while the $\mathrm{X}$-ray survey clearly is an extremely inefficient method to count optical galaxies, with a slope at the bright end near to $2 / 3$, corresponding to about $3 \%$ detections. But it has to be stressed, that our sample in no way forms a complete sample.

Figure 12 shows the $\log N-\log S$ distribution for spiral galaxies and for elliptical plus S0 galaxies for both the $a c$ tive galaxies (upper panel) and for the candidate galaxies sample (lower panel). In the active galaxies sample spirals show up about a factor of 3 more abundant than in the elliptical plus S0 subsample, while this ratio has a value of about 0.8 for the candidate galaxies, showing that optical activity combined with X-ray activity is by far more frequent in spirals. 
Table 2. The reliable high X-ray luminosity sample.

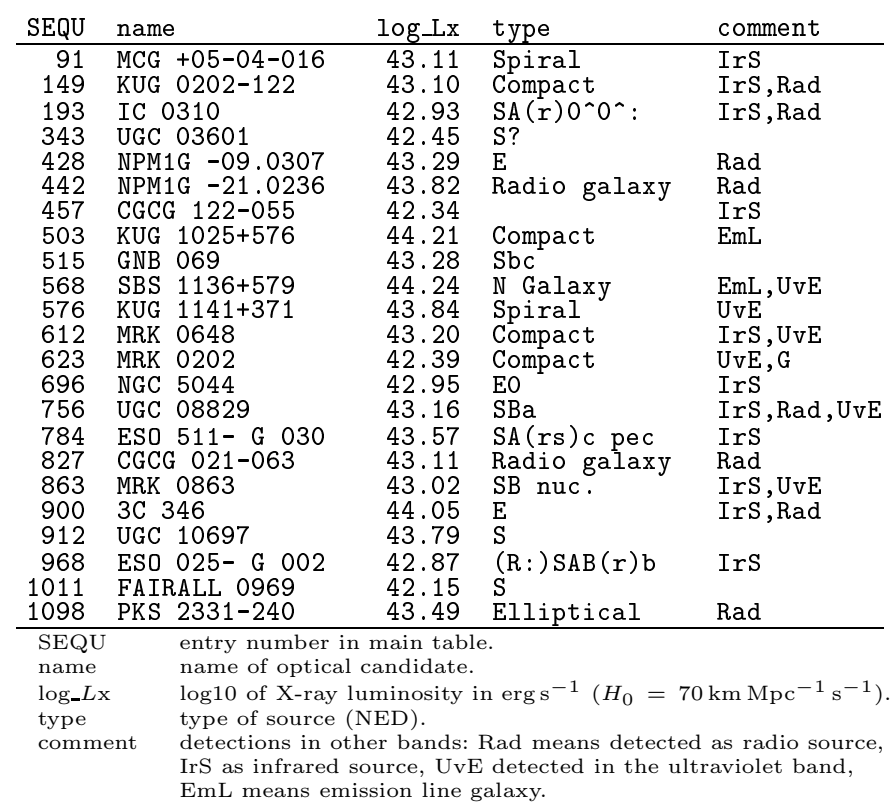

Taking the candidate galaxies sample, we may define a normal galaxy as one where the X-ray to optical luminosity ratio does not exceed $10^{-2}$. As can be seen from Fig. 8 this definition limits the maximum X-ray luminosity of our sample sources to about $10^{42} \mathrm{erg} \mathrm{s}^{-1}$, in agreement with Fabbiano (1989). 97 of the candidate galaxies with known distances have X-ray luminosities $>10^{42} \mathrm{erg} \mathrm{s}^{-1}$, clearly indicating major X-ray activity also in these objects. In a search for normal galaxies remain 43 candidate galaxies with X-ray luminosities $<10^{42} \mathrm{erg} \mathrm{s}^{-1}$ and 58 galaxies with missing distance information as candidates for galaxies that possess no apparent active components in the optical or X-ray regimes.

At the X-ray high luminosity end of the candidate galaxies sample, there are 23 galaxies with reliability being "very good", for which the X-ray luminosity exceeds our limit for a normal galaxy by more than a factor of 10 , that is $\log \left(L_{\mathrm{X}} / L_{\mathrm{opt}}\right)>-1$, and that are not inside or near the line of sight of a group or cluster of galaxies. This high X-ray luminosity sample forms an inhomogeneously studied subsample of galaxies, where a detailled classification will be required to reveal their true nature. As can be seen from Table 2, 11 of the 23 objects are reported also as infrared sources, 8 show radio emission, in 6 cases UV emission has been observed, and 2 are emission line galaxies (NED). For 4 galaxies no detections in other wave bands are reported. The study of the sample is part of a future project. Optical follow-up observations have been initiated.

\section{Conclusions}

In a search for X-ray emission of normal galaxies in the ROSAT All-Sky Survey, we correlated the 18811 RASSBSC sources with the 73197 galaxies from the PGC, with their main parameters updated by the NED database. In 904 cases we detected within a search radius of 100 arcsec around the X-ray source one or more candidates as optical counterparts (the complete sample). A visual screening of optical images overlaid with the observed X-ray contours left finally a sample of 547 optical/X-ray correlations that we regard as sufficiently reliable (reliable correlation sam$p l e)$. For the vast majority of these $\mathrm{X}$-ray emitting galaxies pronounced activity in the optical band (Liners, Seyferts, QSOs, Blazars) has been reported in the literature and the typical X-ray luminosities are lying between $10^{42}$ and $10^{45} \mathrm{erg} \mathrm{s}^{-1}$. For 61 objects data from optical follow up observations (Pietsch et al. 1998; Bischoff et al. 1999) have been used in addition to the NED data to identify and classify the galaxies. The spectral information of the RASS data for the individual galaxies was too scarce to gain additional screening information. There remain 101 sources in our sample as candidates for galaxies with no apparent active components, while a majority of 446 objects definitely belong to the class of objects with active components. This is not surprising, given the fact that sources with luminosities of $10^{40} \mathrm{erg} \mathrm{s}^{-1}$ or less are visible in a typical RASS exposure of order $400 \mathrm{~s}$ only up to distances of about $10 \mathrm{Mpc}$.

Thus correlating X-ray surveys with optical galaxy catalogues is an efficient way to detect galaxies with X-ray active components. In our sample, nuclei both optically and X-ray active, are found predominantly in spirals. Luminosity histograms and $\log N-\log S$ distributions are used in the further characterization of the full sample and different subsamples.

Two special subsamples, interesting for further investigation, are defined: a reliable high X-ray luminosity sample of 23 objects, until now not reported as active nuclei, and a suspected cluster sample of 101 sources where X-ray emission is supposed to originate, at least in parts, from a group or cluster of galaxies.

In addition our work provides soft X-ray parameters, identification maps, and screening results for more than 500 galaxy identifications, useful as a basis for follow-up observations both in the optical and X-ray regimes.

Acknowledgements. The ROSAT project is supported by the Bundesministerium für Bildung und Forschung (BMBF/DLR) and the Max-Planck-Gesellschaft (MPG). This work made use of the NASA/IPAC Extragalactic Database (NED), which is operated by the Jet Propulsion Laboratory, California Institute of Technology, under contract with NASA. The optical images used for the X-ray overlays are taken from the Digitized Sky Survey, that was produced at the Space Telescope Science Institute under US Government grant NAG W-2166. It is based on photographic data of both the National Geographic Society - Palomar Observatory Sky Survey (NGS-POSS) obtained using the Oschin Telescope on Palomar Mountain, and the UK Schmidt Telescope. The NGS-POSS was funded by a grant from the National Geographic Society to the California Institute of Technology. The UK Schmidt Telescope was operated by the Royal Observatory Edinburgh, with funding from 
the UK Science and Engineering Research Council, until 1988 June, and thereafter by the Anglo-Australian Observatory. Original plate material is copyright (c) the Royal Observatory Edinburgh and the Anglo-Australian Observatory.

\section{References}

Bischoff, K., Pietsch, W., Boller, Th., et al. 1999, in Highlights in X-ray Astronomy in honour of Joachim Trümper's 65th birthday, ed. B. Aschenbach, \& M. J. Freyberg, MPE Report, 272, 22

Boese, G., \& Döbereiner, S. 2001, A\&A, 370, 649

Cruddace, R. G., Hasinger, G. R., \& Schmitt, J. H. M. M. 1987, in Astronomy from Large Databases, ed. F. Murtagh, \& A. Heck, ESO Conf. and Workshop Proc., 28, 177

Dickey, J. M., \& Lockman, F. J. 1990, Ann. Rev. Astron. Astrophys., 28, 215

Fabbiano, G. 1989, ARA\&A, 27, 87
Fabbiano, G., Kim, D.-W., \& Trinchieri, G. 1992, ApJS, 80, 531

Hasinger, G., Trümper, J., \& Schmidt, M. 1991, A\&A, 246, 2

Mould, J. R., Huchra, J. P., Freedman, W., et al. 2000, ApJ, 529,786

Paturel, G., Fouqué, P., Bottinelli, L., \& Gouguenheim, L. 1989, A\&AS, 80, 299

Pietsch, W., Bischoff, K., Boller, Th., et al. 1998, A\&A, 333, 48

Trümper, J. 1983, Adv. Space Res., 27, 1404

Voges, W., Gruber, R., Paul, J., et al. 1992, in Proc. European ISY Conf., ed. T. D. Guyenne, \& J. J. Hunt, ESA ISY-3, 223

Voges, W., Aschenbach, B., Boller, T., et al. 1999, A\&A, 349, 389

Walter, R., \& Fink, H. H. 1993, A\&A, 274, 105

Zimmermann, H.-U., Becker, W., Belloni, T., et al. 1997, EXSAS User's Guide, MPE Report, 257 